Eloisa Rosso dos Santos ${ }^{1}$

1. Acadêmica do Curso de Medicina do Hospital Universitário Polydoro Ernani de São Thiago da Universidade Federal de Santa Catarina - UFSC Florianópolis (SC), Brasil (2007/2008).

Recebido do Hospital Universitário Polydoro Ernani de São Thiago da Universidade Federal de Santa Catarina UFSC - Florianópolis (SC), Brasil.

Este trabalho não teve qualquer suporte financeiro.

Submetido em 26 de Agosto de 2009 Aceito em 17 de Dezembro de 2009

Autor para correspondência:

Eloisa Rosso dos Santos

Rua Fernão Dias 165/201 - Centro CEP: 88801-670 - Criciúma (SC), Brasil.

Fone: (48) 8408-6143

E-mail: eloisarosso@gmail.com

\section{Associaçáo do RIFLE com letalidade e tempo de internação em pacientes críticos com lesão renal aguda}

\author{
RIFLE: association with mortality and length of stay in critically \\ ill acute kidney injury patients
}

\section{RESUMO}

Objetivo: Correlacionar a classificação do RIFLE com a letalidade e tempo de internação na unidade de terapia intensiva e no hospital.

Métodos: Estudo de coorte prospectivo, observacional e longitudinal aprovado pelo Comitê de Ética da Instituição. Foram coletados os dados de todos os pacientes internados por mais de 24 horas na unidade de terapia intensiva do Hospital Universitário Polydoro Ernani de São Thiago da Universidade Federal de Santa Catarina de setembro de 2007 a março de 2008 e com seguimento até a alta ou óbito. Os pacientes foram divididos em dois grupos: com lesão renal aguda e sem lesão renal aguda. $\mathrm{O}$ grupo com lesão renal aguda foi classificado conforme o RIFLE e subdividido de acordo com a classe máxima alcançada: risco, injúria ou falência. Não foram incluídas as classes loss e end-stage no estudo. Analisou-se também APACHE II e SOFA. Utilizaram-se os testes $t$ Student e Qui-Quadrado, principalmente. Um $\mathrm{p}<0,05$ foi estatisticamente significativo.

Resultados: A amostra foi composta por 129 pacientes. Desses, 52 (40,3\%) apresentaram lesão renal aguda segundo o RIFLE. Nesse grupo, os doentes foram considerados mais graves obtendo médias maiores de APACHE II e SOFA ( $<<0,05)$. Em comparação ao grupo sem dano renal, a gravidade da lesão renal aguda proporcionou maior letalidade na unidade de terapia intensiva (risco-25\%; injúria-37,5\%; falência-62,5\%) e hospitalar (risco-50\%; injúria-37,5\%; falência-62,5\%) e maior tempo de internação na unidade de terapia intensiva $(\mathrm{p}<0,05)$.

Conclusão: $\mathrm{O}$ sistema RIFLE, conforme a classe de gravidade, foi marcador de risco para maior letalidade na unidade de terapia intensiva e no hospital e maior tempo de internação na unidade de terapia intensiva. Não se encontrou relação para o tempo de internação hospitalar.

Descritores: Insuficiência renal aguda; Unidades de terapia intensiva; Creatinina; Mortalidade hospitalar; Tempo de internação; APACHE

\section{INTRODUÇÁO}

A lesão renal aguda (LRA) é uma condição clínica comum encontrada em pacientes críticos e é reconhecida pelo impacto causado num cenário de terapia intensiva. Entretanto, mesmo com todo esse reconhecimento, há uma falta de um consenso de definição de LRA, ocorrendo mais de 30 conceitos para essa enfermidade. Essa multiplicidade de conceitos é responsável por uma extensa variação na incidência de LRA, em estudos de um único centro $(1-25 \%)^{(1,2-8)}$ e em estudos multicêntricos (39-71\%), ${ }^{(6)}$ e na letalidade (19 a 90\%). ${ }^{(2,3,6,9-20)}$

Para estabelecer uma definição e uma classificação uniforme da LRA, a Acute Dialysis Quality Iniciative (ADQI) ${ }^{(21)}$ desenvolveu, em 2002, o RIFLE (Risco de disfunção, Injúria renal, Falência da função renal, Perda da função renal e Estágio final 
da doença). ${ }^{(2)}$ O RIFLE (Tabela 1) define três graduaçóes de gravidade de disfunção renal - Risco (classe R), Injúria (classe I) e Falência (classe F) baseadas na mudança da creatinina sérica e diurese - e duas classes de evolução (Perda - classe $\mathrm{L}$ - e Estágio Final - classe E).

O surgimento daquele sistema foi fundamental, pois a aplicação de seus critérios possibilitou a confirmação da relação de cada classe do RIFLE com a taxa de letalidade e com o tempo de internação e permitiu a comparação entre os estudos científicos devido a maior uniformização da definição de LRA e a padronização dos critérios que a classificam.

Mais recentemente, uma versão modificada do RIFLE foi proposta pela Acute Kidney Injury Network (AKIN). ${ }^{(22)}$ Quatro modificaçóes foram prontamente identificadas. risco, injúria e falência foram substituídos pelos estágios: 1,2 e 3, respectivamente (Tabela 2); um aumento absoluto na creatinina de ao menos $0,3 \mathrm{mg} / \mathrm{dL}$ foi adicionada ao Estágio 1; pacientes em terapia de substituição renal foram automaticamente classificados em Estágio 3, desconsiderando suas creatinina e diurese; e as categorias Loss e End Stage foram eliminadas.

Após a publicação do RIFLE em 2004 e do AKIN em 2007, diversas investigaçôes foram publicadas utilizando essas classificaçóes. ${ }^{(1,9,10,23-34)}$ Cruz et al., ${ }^{(31)} \mathrm{em}$ seu artigo de re- visão, reconhecem que nenhuma classificação será perfeita e que o próximo passo seria reconciliar as definiçóes existentes, movendo a comunidade médica em direção a um consenso de definição. Para isso o autor infere que mais estudos prospectivos revelam-se essenciais para destacar toda a capacidade dos critérios já estabelecidos. Dessa maneira, o objetivo do presente estudo foi correlacionar prospectivamente a classificação de RIFLE com a letalidade e com o tempo de internação na unidade de terapia intensiva (UTI) e no hospital naqueles pacientes admitidos em uma UTI geral.

\section{MÉTODOS}

\section{Delineamento do estudo}

Estudo de coorte prospectiva, observacional e longitudinal, no qual foram coletados os dados de todos os pacientes admitidos na UTI do Hospital Universitário Polydoro Ernani de São Thiago da Universidade Federal de Santa Catarina (HU-UFSC) no período de setembro de 2007 a março de 2008, tendo sido aprovado pelo Comitê de Ética em Pesquisas com Seres Humanos da UFSC e executado de acordo com a Declaração de Helsinki. Incluíram-se no estudo todos os pacientes que concordaram em participar da pesquisa e

Tabela 1 - Classificaçáo proposta para lesão renal aguda - RIFLE

\begin{tabular}{|c|c|c|}
\hline Classificação RIFLE & Critério TFG & Critério diurese \\
\hline Risk & $\begin{array}{c}\text { aumento da SCr x 1,5 } \\
\text { ou diminuiçáo da TFG > 25\% }\end{array}$ & diurese $<0,5 \mathrm{~mL} / \mathrm{Kg} / \mathrm{h}$ em $6 \mathrm{~h}$ \\
\hline Injury & $\begin{array}{l}\text { aumento da SCr x } 2 \\
\text { ou diminuição da TFG > 50\% }\end{array}$ & diurese $<0,5 \mathrm{~mL} / \mathrm{Kg} / \mathrm{h}$ em $12 \mathrm{~h}$ \\
\hline Failure & $\begin{array}{c}\text { aumento da SCr x } 3 \\
\text { ou diminuição da TFG > 75\% ou SCr }>4 \mathrm{mg} / \mathrm{dL}\end{array}$ & $\begin{array}{c}\text { diurese }<0,3 \mathrm{~mL} / \mathrm{Kg} / \mathrm{h} \text { em } 24 \mathrm{~h} \\
\text { ou anúria por } 12 \mathrm{~h}\end{array}$ \\
\hline Loss & $\begin{array}{l}\text { perda completa da função renal } \\
\qquad>4 \text { semanas }\end{array}$ & \\
\hline End-stage kidney disease & $\begin{array}{c}\text { necessidade de TSR } \\
>3 \text { meses }\end{array}$ & \\
\hline
\end{tabular}

RIFLE - Risk Injury Failure Loss End; TFG - taxa de filtração glomerular; SCr - creatinina sérica; TSR - terapia de substituição renal.

Adaptado de: Bellomo R, Ronco C, Kellum JA, Mehta RL, Palevsky P, Acute Dialysis Quality Initiative workgroup. Acute renal failure - definition, outcome measures, animal models, fluid therapy and information technology needs: the Second International Consensus Conference of the Acute Dialysis Quality Iniciative (ADQI) Group. Crit Care. 2004;8(4):R204-12.

Tabela 2 - Classificaçáo AKIN

\begin{tabular}{lcc}
\hline Classificação RIFLE & Critério TFG & Critério diurese \\
\hline Stage 1 & aumento da SCr x 1,5 & diurese $<0,5 \mathrm{~mL} / \mathrm{Kg} / \mathrm{h} \mathrm{em} 6 \mathrm{~h}$ \\
Stage 2 & ou $>0,3 \mathrm{mg} / \mathrm{dL}$ & diurese $<0,5 \mathrm{~mL} / \mathrm{Kg} / \mathrm{h} \mathrm{em} 12 \mathrm{~h}$ \\
Stage 3 & aumento da SCr x & diurese $<0,3 \mathrm{~mL} / \mathrm{Kg} / \mathrm{h} \mathrm{em} 24 \mathrm{~h}$ \\
& aumento da SCr x 3 ou aúria por $12 \mathrm{~h}$ \\
& ou SCr $>4 \mathrm{mg} / \mathrm{dL}$ & \\
\hline
\end{tabular}

TFG - Taxa de filtração glomerular; SCr - creatinina sérica.

Adaptado de: Mehta RL, Kellum JA, Shah SV, Molitoris BA, Ronco C, Warnock DG, Levin A; Acute Kidney Injury Network. Acute Kidney Injury Network: report of an initiative to improve outcomes in acute kidney injury. Crit Care. 2007;11(2):R31. 
que assinaram o TCLE. Na impossibilidade de consentimento por parte do paciente, apresentou-se o TCLE ao responsável legal. Foi considerada somente a primeira admissão para aqueles indivíduos readmitidos na UTI no período analisado.

\section{Critérios de exclusão}

Foram excluídos do estudo os pacientes com idade inferior a 16 anos; com morte encefálica clínica diagnosticada no momento da internação na UTI ou que tiveram esse diagnóstico até seis horas após a internação; com insuficiência renal em tratamento dialítico, antes da internação na UTI; transferidos de outra UTI e que nessa permaneceram internados por mais que 24 horas; que náo completaram 24 horas de internação na UTI do HU-UFSC e que se recusaram a assinar o Termo de Consentimento Livre e Esclarecido (TCLE).

\section{Procedimentos}

O estudo foi realizado durante o período de internação de cada paciente sendo somente acompanhada a evoluçáo do paciente, coletado os dados a partir do prontuário do mesmo, conforme um protocolo, e procedido à classificação de acordo com o RIFLE durante sua internaçáo na UTI. Cada paciente foi seguido até o seu destino final, alta ou óbito, sendo registradas as respectivas datas para as contagens do tempo de internação na UTI e no hospital.

Foram avaliados parâmetros clínicos fisiológicos (Acute Physiology and Chronic Health Evaluation II - APACHE II ${ }^{(35)}$ e Sequential Organ Failure Assessment - SOFA ${ }^{(36)}$ ) e laboratoriais (creatinina sérica - $S_{\text {cr }}$ antes da internação na UTI e durante a mesma). Os parâmetros fisiológicos foram coletados a fim de avaliar a gravidade da enfermidade de cada paciente. O APACHE II foi coletado após o paciente ter completado 24 horas de internação na UTI. Já o escore SOFA foi coletado no instante da admissão na UTI e, depois, quando o paciente concluía 24 horas, três dias, cinco dias e sete dias de internação na UTI. Foi calculado também o SOFA médio de todos os pacientes que tiveram mais de um SOFA. Este cálculo foi realizado através de uma média aritmética simples.

$\mathrm{O}$ pesquisador classificou os pacientes de acordo com a classe máxima do RIFLE alcançada durante a internaçáo na UTI. Por exemplo, o paciente que teve injúria renal (classe I) na admissão da UTI e durante a internação desenvolveu falência da funçáo renal (classe F) foi classificado como tendo falência $(F)$. Muitos pacientes com disfunção renal, identificada no momento da admissão na UTI, não tinham o valor da $\mathrm{S}_{\mathrm{Cr}}$ medido anteriormente. Isso representaria um problema para o sistema do RIFLE que considera a mudança proporcional daquele valor. Assim, na falta da $S_{\mathrm{Cr}}$ antes da internação, a mesma foi estimada pelo cálculo da equação simplificada do estudo de Modificação da Dieta em Doenças Renais
(MDRD) $)^{(37)}$ conforme definido na publicação original do RIFLE e recomendado pela ADQI.(2) Para resolver a equação do MDRD, assumiu-se a taxa de filtração glomerular (TFG) de $75 \mathrm{~mL} /$ minuto $/ 1,73 \mathrm{~m}^{2}$. As classes do RIFLE foram determinadas baseadas na pior das variáveis, as quais dependeram da mudança proporcional da creatinina sérica medida laboratorialmente ou estimada pelo cálculo da MDRD. Dessa forma, os pacientes que se enquadraram na classificação do RIFLE tiveram o diagnóstico de LRA. O grupo com LRA foi subdividido em R (Risco), I (Injúria) e F (Falência). Nesse estudo, não foram avaliadas as classes L e E do RIFLE.

\section{Análise estatística}

Foram realizadas estatísticas descritivas e analíticas através de porcentagem simples e medidas de ocorrência e de associação (risco relativo - RR - com intervalo de confiança - IC - de 95\%). Foi empregada, como tendência central, a média com + desvio-padrão (+ DP) e a mediana para observaçóes contínuas. As variáveis qualitativas foram analisadas através do teste não-paramétrico de Qui-Quadrado, sendo considerado estatisticamente significativo quando $\mathrm{p}<0,05$. Ao encontrar um valor esperado menor que cinco na célula da tabela de contingências, usou-se o teste exato de Fisher. As variáveis quantitativas foram comparadas pelo teste paramétrico t Student e quando essas possuíam variâncias populacionais desconhecidas foi adotado o teste F. A significância estatística desses dois últimos testes foi considerada quando $\mathrm{p}<0,05$.

A análise multivariada foi realizada através de regressão de Cox para controle das variáveis independentes. A variável "tempo" utilizada na regressão foi o tempo de internaçáo hospitalar. Pela colinearidade existente entre as variáveis sepse e choque séptico, seguindo o princípio da multicolinearidade, foi somente utilizada a última variável.

A curva de Kaplan-Meier foi construída para estimar a probabilidade de sobrevida, em relação ao tempo de internação hospitalar, conforme a presença de LRA e de acordo com o RIFLE máximo (R, Ie F). Foi usado um IC de 95\% censurando os pacientes que receberam altas. Para essa estimativa, utilizou-se o teste de log-rank, tendo significância estatística quando $\mathrm{p}<0,05$.

\section{RESULTADOS}

Durante o intervalo estudado, internaram-se na UTI 198 pacientes. Desses, 69 foram excluídos: um por ter idade inferior a 16 anos, cinco por já apresentarem insuficiência renal em tratamento dialítico antes da admissão na UTI, três por terem sido transferidos de outra UTI e permanecido nessa UTI por mais que 24 horas, 57 por não completarem 24 horas de internação na UTI, dois por não concordarem com 
o TCLE e um por fuga hospitalar. Portanto, 129 pacientes compuseram a coorte desse trabalho.

As características clínicas e demográficas da população estudada estão representadas na tabela 3 através de análise univariada. O grupo sem LRA foi composto por 77 (59,7\%) pacientes e o grupo com LRA por 52 (40,3\%). A incidência de LRA, de acordo com a classe máxima do RIFLE alcançada, foi de 15,5\%, 12,4\% e 12,4\% para R, Ie F, respectivamente (Tabela 4).

A progressão da LRA, durante a internação na UTI, para a classe máxima do RIFLE é mostrada na figura 1 . No primeiro dia de admissão, vinte e cinco pacientes $(19,4 \%)$ já tinham LRA definida pelos critérios do RIFLE. Destes, quatro
$(3,1 \%)$ já estavam na classe F. Durante toda a internaçáo na UTI, a contar do dia de admissão, cinqüenta e dois pacientes $(40,3 \%)$ tiveram um episódio de LRA pertencendo ao grupo com LRA. Sete pacientes $(5,4 \%)$ com classe $\mathrm{R}$ progrediram para RIFLE classe I (1,5\%) ou F (3,9\%). Já entre aqueles pacientes que pertenciam à classe I na admissão, somente um $(0,8 \%)$ desenvolveu falência renal. Houve pacientes que após enquadrarem-se na classe $\mathrm{R}(6,2 \%)$ ou na classe I $(3,9 \%)$ permaneceram nessas até o destino final, alta ou óbito. Dos 104 pacientes $(80,6 \%)$ que tinham a função preservada no primeiro dia de admissão, $12(9,3 \%)$ progrediram para a classe $\mathrm{R}$, nove $(7 \%)$ para I e seis $(4,6 \%)$ para a F.

Tabela 3 - Análise univariada das características clínicas e demográficas dos pacientes com e sem lesão renal aguda

\begin{tabular}{|c|c|c|c|c|c|c|}
\hline & Sem LRA & & Com LRA & & Risco relativo & \\
\hline Características & $\mathrm{N}$ & $\%$ & $\mathrm{~N}$ & $\%$ & (IC 95\%) & Valor de p \\
\hline $\mathrm{N}$ & 77 & 59,7 & 52 & 40,3 & - & - \\
\hline Média + DP & $46,8+16,5$ & & $56,4+18,8$ & & - & $0,0028^{*}$ \\
\hline Sexo & & & & & & NS \\
\hline Feminino & 36 & 46,8 & 24 & 46,2 & $1,01(0,67-1,55)$ & \\
\hline Raça & & & & & & NS \\
\hline Branca & 68 & 88,3 & 46 & 88,5 & $0,99(0,51-1,92)$ & \\
\hline Negra & 9 & 11,7 & 6 & 11,5 & & \\
\hline \multicolumn{7}{|l|}{ Origem do paciente $^{\dagger}$} \\
\hline Emergência & 35 & 45,5 & 17 & 32,7 & - & NS \\
\hline Outro hospital & 15 & 19,5 & 8 & 15,4 & - & NS \\
\hline Causa da admissão & & & & & & NS \\
\hline Clínica & 49 & 63,6 & 33 & 63,5 & $1,00(0,65-1,55)$ & \\
\hline Cirúrgica & 28 & 36,4 & 19 & 36,5 & & \\
\hline Sepse & & & & & & $0,012 \ddagger$ \\
\hline Sim & 3 & 3,9 & 9 & 17,3 & $2,04(1,36-3,06)$ & \\
\hline Não & 74 & 96,1 & 43 & 82,7 & - & \\
\hline Choque séptico & & & & & & $<0,05 \$$ \\
\hline Sim & 5 & 6,5 & 10 & 19,2 & $1,81(1,18-2,78)$ & \\
\hline Não & 72 & 93,5 & 42 & 80,8 & - & \\
\hline
\end{tabular}


Tabela 4 - Associaçáo dos escores de gravidade do paciente (APACHE II, SOFA admissáo e SOFA médio) e resultados (letalidade e tempo de internaçáo) com o grupo sem lesão renal aguda e os diferentes subgrupos do RIFLE

\begin{tabular}{|c|c|c|c|c|c|}
\hline & $\begin{array}{c}\text { Sem LRA } \\
\mathrm{N}=77\end{array}$ & $\begin{array}{l}\text { Risco } \\
\mathrm{N}=20\end{array}$ & $\begin{array}{l}\text { Injúria } \\
\mathrm{N}=16\end{array}$ & $\begin{array}{l}\text { Falência } \\
\mathrm{N}=16\end{array}$ & Valor de $\mathrm{p}$ \\
\hline$\%$ & 59,7 & 15,5 & 12,4 & 12,4 & \\
\hline APACHE II & & & & & $<0,0000^{*}$ \\
\hline Média + DP & $14,5+4,9$ & $18,5+5,8$ & $19,0+5,8$ & $23,0+5,2$ & \\
\hline Mediana & 14 & 18,5 & 17,5 & 23,5 & \\
\hline SOFA (admissão) & & & & & $<0,0000^{*}$ \\
\hline Média + DP & $2,4+2,3$ & $2,9+1,8$ & $3,9+2,1$ & $7,0+3,0$ & \\
\hline Mediana & 2 & 3 & 3,5 & 7 & \\
\hline SOFA médio & & & & & $<0,0000^{*}$ \\
\hline Média + DP & $3,5+2,3$ & $5,5+3,0$ & $5,7+3,2$ & $8,5+2,5$ & \\
\hline Mediana & 3 & 5 & 5,3 & 8,8 & \\
\hline Letalidade UTI & & & & & $<0,00001 \dagger$ \\
\hline$\%$ & 7,8 & 25 & 37,5 & 62,5 & \\
\hline RR (IC 95\%) & $0,19(0,10-0,35)$ & $1,24(1,14-1,34)$ & $2,07(1,58-2,71)$ & $4,16(2,45-7,04)$ & \\
\hline Letalidade hospitalar & & & & & $<0,00001 \dagger$ \\
\hline$\%$ & 10,4 & 50 & 37,5 & 62,5 & \\
\hline RR (IC 95\%) & $0,20(0,11-0,36)$ & $2,27(1,67-3,07)$ & $1,51(1,29-1,75)$ & $2,97(1,98-4,44)$ & \\
\hline $\begin{array}{l}\text { Tempo internação UTI horas } \\
\text { (Média + DP) }\end{array}$ & $127,7+133,05$ & $186,8+177,66$ & $244,5+180,71$ & $312,7+386,30$ & \\
\hline $\begin{array}{l}\text { Tempo internação hospitalar } \\
\text { horas (Média + DP) }\end{array}$ & $572,8+650,09$ & $655,2+716,67$ & $826,5+882,03$ & $603,0+599,64$ & \\
\hline
\end{tabular}

LRA - lesão renal aguda; UTI - unidade de terapia intensiva; DP- desvio-padrão; NS - náo significativo; RR - risco relativo; IC- intervalo de confiança; APACHE - Acute Physiologic Chronic Health Evaluation; SOFA - Sequential Organ Failure Assessment; RIFLE - Risk Injury Failure Loss End. *Associação isolada de cada escore através do teste t Student (nível de significância $<5 \%$ ou $\mathrm{p}<0,05$ ). ${ }^{\dagger}$ Associação entre as subdivisóes do RIFLE com a taxa de letalidade na UTI utilizando o teste de Qui-Quadrado ( $\mathrm{p}<0,05$, estatisticamente significante). ${ }^{\ddagger}$ Associação utilizando o teste F ( $\mathrm{p}<$ 0,05, significante estatisticamente).

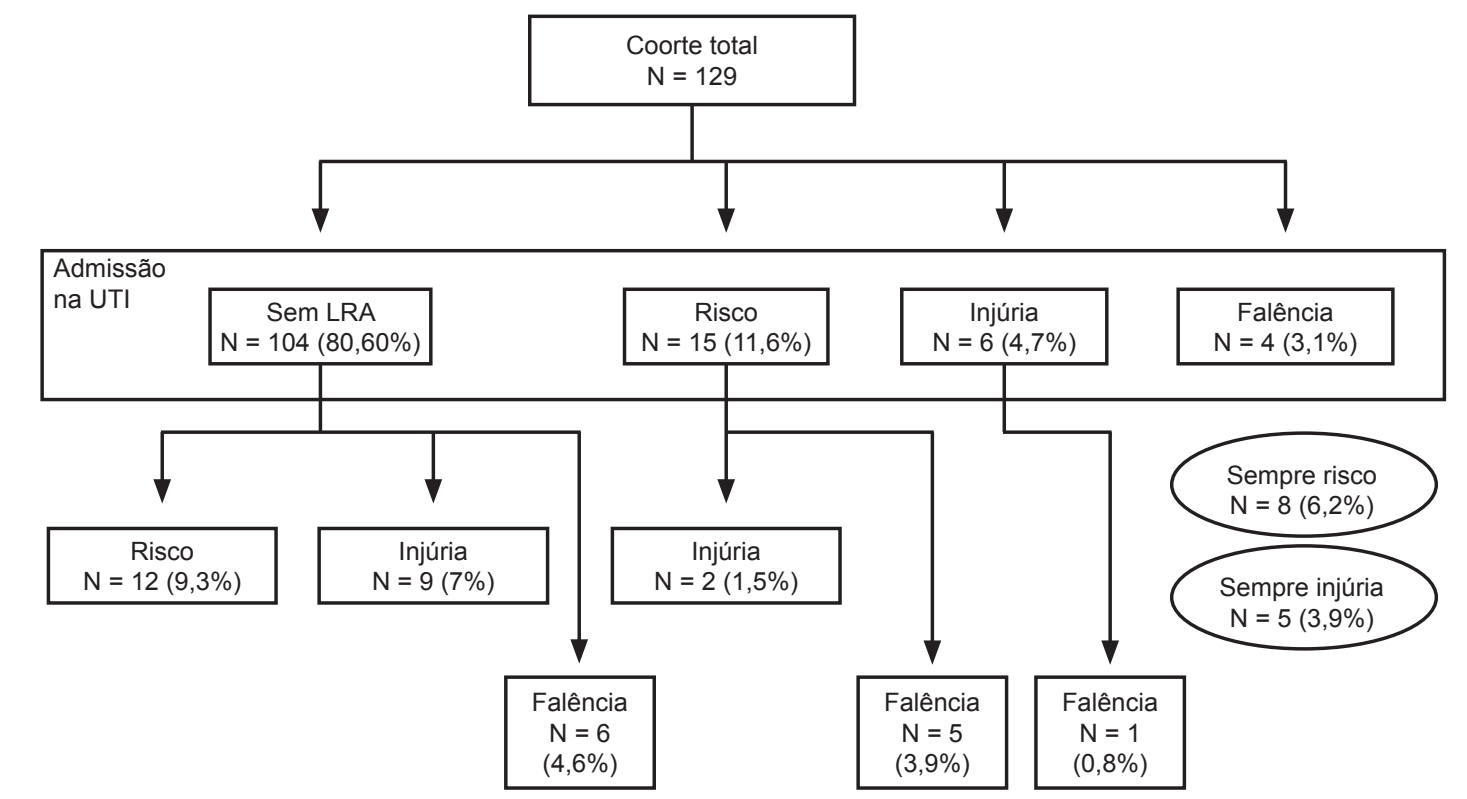

Totais: risco máximo 20 (15,5\%); injúria máxima 16 (12,4\%); falência máxima 16 (12,4\%)

Os dados expressam o número de pacientes que foram identificados em cada nível e a percentagem do número total do estudo. "Sempre risco" e "Sempre injúria" foram aqueles pacientes que durante a admissão estavam assim classificados e não progrediram para a classe posterior. UTI - unidade de terapia intensiva; LRA - lesão renal aguda.

Figura 1 - Fluxograma do curso clínico dos pacientes até a classe máxima do RIFLE. 
Observa-se na tabela 4 que as médias do APACHE II foram mais altas nos subgrupos com LRA de acordo com o RIFLE ( $p<0,0000)$. Igualmente, isso foi verificado no SOFA da admissão e médio com alto nível de significância estatísti$\mathrm{ca}(\mathrm{p}<0,0000)$. Além disso, as medianas também foram mais altas nos subgrupos com LRA.

A gravidade progressiva da LRA, de acordo com o RIFLE para os subgrupos R, I e F, foi associada com aumento da taxa de letalidade na UTI (Tabela 4) bem como com maior tempo de internaçáo nesse local (Tabela 4). Percebeu-se ainda que quanto maior a classificação do RIFLE alcançada, em associação à letalidade na UTI, maior foi o risco relativo do paciente a ela enquadrado (Tabela 4). Em relação à taxa de letalidade hospitalar (Tabela 4), notou-se que o subgrupo I, embora representasse uma classe mais grave em comparação ao $\mathrm{R}$, apresentou menor taxa de letalidade que esse subgrupo. Já o F continuou a demonstrar a maior taxa de letalidade hospitalar, assim como foi observado na taxa de letalidade na UTI. O subgrupo F mostrou maior RR para letalidade hospitalar $(2,97 ; \mathrm{p}<0,00001)$, igualmente ao obtido para a letalidade na UTI. No entanto, as classes menos graves, R e I não seguiram o mesmo padrão de aumento no $R R$ conforme a gravidade de $L R A(R R=2,27$ para $R$ e $R R=1,51$ para $\mathrm{I}$ ).

Os pacientes do grupo com LRA ficaram mais tempo, em média, internados na UTI (Tabela 4). A comparação entre as médias do tempo de internação hospitalar (Tabela 4), para cada um dos subgrupos do grupo com LRA, não mostrou significância estatística.

$\mathrm{Na}$ análise multivariada (Tabela 5), os fatores associados independentemente à letalidade hospitalar foram o uso de droga vasoativa (DVA) e o APACHE II. Os pacientes com uso de DVA apresentaram um risco de óbito cinco vezes maior

Tabela 5 - Análise multivariada de fatores associados à mortalidade hospitalar

\begin{tabular}{lcccc}
\hline Variáveis & RP & HR & IC & Valor \\
Bruta & Ajustada & $95 \%$ & de p \\
\hline Idade $>30$ anos & 3,12 & 1,02 & $1,01-1,05$ & 0,015 \\
Choque séptico & 2,03 & 1,84 & $0,62-5,51$ & 0,2728 \\
$\begin{array}{l}\text { Ventilaçáo mecânica } \\
\text { Droga vasoativa }\end{array}$ & 4,06 & 2,89 & $0,64-14,03$ & 0,1635 \\
APACHE & 7,04 & 5,35 & $1,70-16,83$ & 0,004 \\
$\quad 18-24$ & 2,92 & 2,96 & $1,15-7,64$ & 0,0241 \\
$\quad>25$ & 5,56 & 3,11 & $1,02-9,48$ & 0,0452 \\
SOFA & & 0,92 & $0,78-1,09$ & 0,3286 \\
RIFLE & 1,81 & 1,5 & $0,58-3,92$ & 0,4056 \\
\hline R
\end{tabular}

RP- razão de prevalência bruta; HR - hazard ratio ajustada; IC - intervalo de confiança; APACHE - Acute Physiologic Chronic Health Evaluation; SOFA - Sequential Organ Failure Assessment; RIFLE - Risk Injury Failure Loss End.
(HRaj=5,35) do que aqueles que não utilizaram. Já aqueles com APACHE II acima de 25 tiveram mais que o triplo de óbitos comparados aos níveis abaixo de 18 deste índice.

$\mathrm{Na}$ curva de Kaplan-Meier (Figura 2), os indivíduos do grupo sem LRA tiveram uma probabilidade de sobrevida maior ( $\mathrm{p}=0,0002)$, principalmente, quando comparados ao subgrupo $\mathrm{F}$. Na comparação entre as quatro curvas, o teste de log-rank mostrou um $\mathrm{p}=0,0002$, sendo significativo estatisticamente.

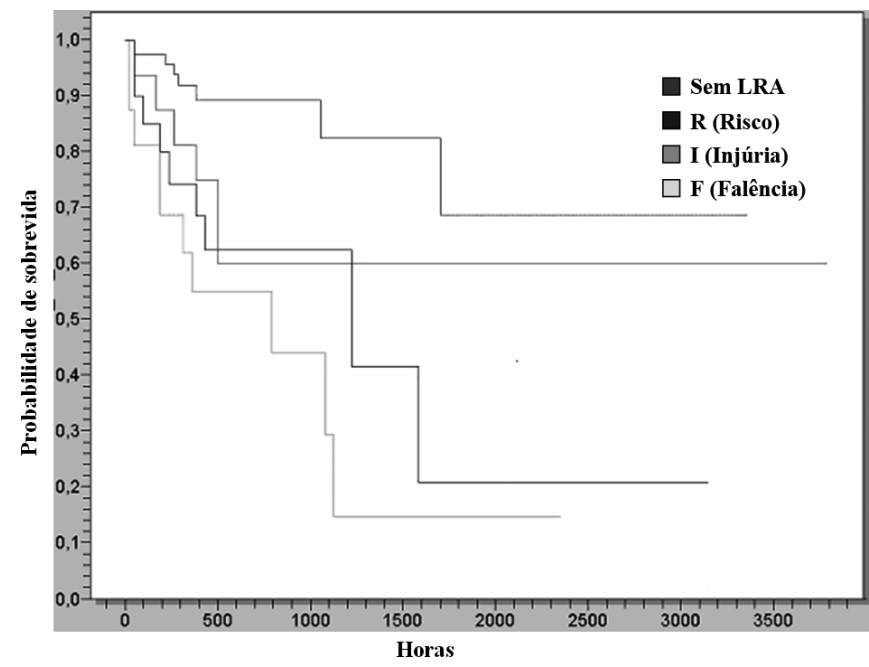

Pacientes que receberam alta hospitalar foram censurados. Estatística de Log-Rank, $\mathrm{p}=0,0002$.

LRA - lesão renal aguda. Horas = tempo de internação hospitalar.

Figura 2 - Curva de Kaplan-Meier para probabilidade de sobrevida (hospitalar) conforme a presença de LRA de acordo com o RIFLE máximo R, I e F.

\section{DISCUSSÁO}

Ao verificar a incidência da LRA, definida pelo critério do RIFLE no presente estudo, encontrou-se um valor de 40,3\%. Maccariello et al., ${ }^{(23)}$ Bell et al. ${ }^{(24)}$ e Abosaif et al. ${ }^{(25)}$ não obtiveram a incidência de LRA em seus estudos, uma vez que utilizaram somente pacientes com LRA. Hoste et al. ${ }^{(9)}$ encontrou uma incidência de 67,2\%. Ostermann e Chang ${ }^{(10)}$ mostraram que $35,8 \%$ dos seus pacientes tiveram LRA. As possíveis explicações para as variações encontradas entre as incidências são várias. Primeiro, alguns dos estudos citados são retrospectivos e contam com prontuários do arquivo médico nos quais, muitas vezes, faltam dados, podendo, assim, enviesar a determinação da doença. Segundo, seria que Hoste et al. ${ }^{(9)}$ e Ostermann e Chang ${ }^{(10)}$ utilizaram dados dos anos de 2000 a 2001 e de 1989 a 1999, respectivamente. É possível que a incidência da LRA, no decorrer dos anos até o presente momento, tenha mudado verdadeiramente. Outras explicaçóes seriam os diferentes tipos de UTI e de pacien- 
tes estudados, por exemplo: pacientes sépticos, queimados, pacientes com LRA em diálise. Conforme Uchino et al., ${ }^{(6)}$ houve uma incidência significantemente maior de LRA em UTIs específicas versus UTIs gerais, bem como grandes UTIs versus pequenas unidades.

Verificou-se que os pacientes cuja classe apresentada era I já na admissão pouco progrediram para a classe F. Ao contrário dos pacientes que apresentaram classe $\mathrm{R}$ na admissão, dos quais, aproximadamente, $50 \%$ avançaram para classe I ou F. Isso pode ser explicado pelo fato de que pacientes em classe I, em relação à classe $\mathrm{R}$, não só apresentavam indícios laboratoriais de LRA como também clínicos. Assim, por essa última condição ser mais evidente, a terapêutica pôde ter sido iniciada mais precocemente evitando-se a evoluçáo da doença. Segundo Hoste et al.,(9) mais que 50\% dos pacientes em risco (R), em seu estudo, progrediram para classe I ou F. Esse achado está em concordância com o presente estudo. Porém, o mesmo autor relatou que mais de um terço dos pacientes com classe I progrediram para classe $\mathrm{F}$, diferindo do estudo em questáo, cuja progressão de uma classe para a outra foi de um sexto.

Esperava-se também que os altos escores, tanto do SOFA quanto do APACHE II, estivessem correlacionados com a incidência de LRA. De acordo com o esperado, a associaçáo isolada, de cada um daqueles escores com a presença de LRA, mostrou que as médias e as medianas mais altas pertenciam ao grupo com LRA. Percebeu-se, entáo, que os pacientes que se enquadraram no RIFLE, com LRA, tinham um maior grau de gravidade de doença tanto em relação ao APACHE II, encontrado nas primeiras 24 horas, quanto ao SOFA da admissão e médio. Essa mesma associação foi apresentada por Abosaif et al. ${ }^{(25)}$ e por Hoste et al. ${ }^{(9)}$ Além disso, os altos escores do APACHE II estavam diretamente correlacionados a um maior risco de óbito hospitalar.

$\mathrm{Na}$ tentativa de validar o RIFLE como preditor de prognóstico dos pacientes da UTI, foi analisada a associação de cada subgrupo R, I e F com a letalidade na UTI e no hospital bem como com o tempo de internaçáo. Dessa forma, demonstrouse que quanto mais grave era a classe do RIFLE maior era a letalidade na UTI e que quando se comparava o grupo com LRA com o grupo sem LRA, esse último mostrava uma taxa de letalidade ainda menor. A letalidade hospitalar não obteve o mesmo padrão de aumento da letalidade conforme a gravidade do RIFLE, todavia, o subgrupo F continuou com a maior taxa. Bell et al. ${ }^{(24)}$ mostrou que $57,9 \%$ dos pacientes da classe F morreram em 30 dias comparados com $23,5 \%$ da classe R e $22 \%$ da classe I. Abosaif et al. ${ }^{(25)}$ apresentou taxa de letalidade na UTI de $16,7 \%$ para pacientes do grupo controle e $38,3 \%$, $50 \%$ e $74,5 \%$ para aqueles do grupo: Risco, Injúria e falência. Hoste et al..$^{(9)}$ encontrou em seu estudo taxas de letalidade hospitalar de 8,8\%, 11,4\% e 26,3\% para aqueles mesmos subgrupos comparados com 5,5\% dos pacientes com função renal preservada. Uchino et al. ${ }^{(1)}$ obteve taxas de $4,4 \%$ para indivíduos sem LRA e 15,1\%, 29,2\% e 41,1\% para as classes R, I e F. Lopes et al. ${ }^{(27)}$ aplicaram os critérios do RIFLE em pacientes queimados e achou taxas de letalidade de 11,1\%, 63,6\% e $75 \%$ de acordo com a gravidade da LRA contra $6 \%$ para pacientes sem LRA. Ostermann e Chang ${ }^{(10)}$ exibiram uma taxa de letalidade de 27,9\% para pacientes sem LRA e 19\%, 26,6\% e 22,98\% para aqueles com R, I e F. Para essas mesmas classes, Maccariello et al. ${ }^{(23)}$ estabeleceu taxas de 72\%, 79\% e 76\%, mas não conseguiu significância estatística para essa associação. Em 2007, Lopes et al. aplicaram o RIFLE em pacientes infectados com o HIV ${ }^{(28)}$ e mostrou taxas de letalidade de $23,5 \%$ para $\mathrm{R}, 66,6 \%$ para I, $72 \%$, para F e $23,5 \%$ para indivíduos sem LRA. No mesmo ano, porém em pacientes com sepse, Lopes et al. ${ }^{(29)}$ encontraram taxas de $27,3 \%$ (R), 28,6\% (I), 55\% (F) e 9,6\% (sem LRA). Apesar das porcentagens variarem nos diversos estudos e entre eles e o presente estudo, nota-se que as taxas de letalidade seguem uma mesma direçáo, sendo que as maiores correspondem à classe mais grave do RIFLE.

Ainda em relação à letalidade na UTI e no hospital, observou-se que a exposição à LRA, de acordo com o RIFLE, representou risco para a letalidade naqueles locais e que quanto mais grave era a classe alcançada maior o risco inerente a ela. Outros estudos ${ }^{(1,9,10,27)}$ encontraram essa mesma associação. Bell et al. ${ }^{(24)}$ acharam associação somente para a classe $\mathrm{F}$. Lopes et al. ${ }^{(28)}$ mostraram, igualmente, associação para uma única classe, a I. Houve aqueles que não conseguiram associar o RIFLE com a letalidade. A exemplo, tem-se Maccariello et al. ${ }^{(23)}$ o qual mesmo apresentando odds ratios de 1,47 e 1,19 para a classe I e $\mathrm{F}$, respectivamente, não puderam confirmálos pelo IC além de não ter tido significância estatística. O motivo para essa discordância supóe-se estar nas populaçóes escolhidas para esses estudos, uma vez que Bell et al. ${ }^{(24)} \mathrm{e}$ Maccariello et al. ${ }^{(23)}$ utilizaram pacientes que já apresentavam LRA em tratamento dialítico e Lopes et al. ${ }^{(28)}$ alocaram em sua amostra somente pacientes HIV positivos. Por outro lado, ao rever a análise multivariada, o RIFLE náo esteve independentemente associado à letalidade hospitalar.

Analisando as médias do tempo de internação na UTI, percebeu-se que, igualmente à letalidade, elas eram maiores nas classes mais graves da LRA e que quando se tinha a classe menos grave, $\mathrm{R}$, essa também era maior quando comparada aos pacientes com função renal preservada. Dessa maneira, encontrou-se associaçấo entre o RIFLE e um maior tempo de internação na UTI. Hoste et al. ${ }^{(9)}$ e Ostermann e Chang ${ }^{(10)}$ encontraram essa mesma relação. Outros estudos, já citados, não mencionam esse tipo de associação. Semelhantemente, tentouse associar o RIFLE ao tempo de internaçáo hospitalar, no entanto, não se encontrou diferença estatisticamente significativa 
no presente estudo, apesar de o tempo médio ser maior em todas as classes de LRA em relaçáo ao grupo sem LRA.

Evidentemente, o presente estudo tem suas limitaçóes. A principal delas é o tempo que foi dedicado à pesquisa, uma vez que um período de seguimento mais prolongado daria uma amostra maior e, possivelmente, permitiria uma análise mais robusta. Outra limitação, não menos importante, foi a utilização de um único centro de pesquisa. Isso incapacitou, de certa forma, a generalização dos resultados dando pouca validade externa ao trabalho. Sabe-se também que o fato de náo se ter usado a diurese do paciente, para que se comparasse com o critério da creatinina, trouxe prejuízo ao estudo. É possível que a diurese e creatinina juntas fornecessem informaçóes complementares. A fim de amenizar essa lacuna, utilizou-se a creatinina de acordo com a proposta da ADQI.

A ADQI recomendou, igualmente, o uso da equação da MDRD como alternativa para a falta da $S_{\mathrm{Cr}}$ Porém, o emprego dessa determinação prejudicou, em parte, o estudo, pois, sendo essa equação uma estimativa da creatinina, pôde ter-se superestimado a incidência e a gravidade da LRA. Embora a equação da MDRD tenha sido desenvolvida e validada em um grande número de pacientes, resultados conflituosos foram publicados a esse respeito. Reconhece-se que a $S_{\mathrm{Cr}_{\mathrm{r}}}$ calculada não substitui a creatinina real, mas a validação da MDRD ou o desenvolvimento de uma alternativa esteve longe do objetivo desse estudo.

Além disso, podem ter ocorrido vieses no estudo, principalmente, pela distribuiçáo heterogênea de algumas características entre as categorias do RIFLE (idade, comorbidades, sepsis, uso de DVA e ventilação mecânica). Por esse motivo, empregou-se a análise multivariada para ajuste das variáveis em relação à letalidade hospitalar.

Por outro lado, salienta-se que sendo prospectivo o presente estudo dispóe de maior credibilidade e precisão às associações nele realizadas. Ademais, todos os resultados foram observaçóes reais baseadas no acompanhamento diário dos pacientes. Além disso, o presente estudo é um dos poucos trabalhos brasileiros com um desenho prospectivo aplicado em uma UTI geral o que o torna uma avaliação razoavelmente real do dia-a-dia dos cuidados da Terapia Intensiva.

\section{CONCLUSÁO}

Tem-se que o uso do RIFLE foi um marcador de risco para uma maior letalidade na UTI e no hospital, conforme a gradaçáo da gravidade da LRA. Para o tempo de internação, o RIFLE foi igualmente um marcador de maior tempo de internação na UTI, segundo a gravidade progressiva da LRA. $\mathrm{O}$ mesmo não foi encontrado para o tempo de internaçáo hospitalar.
Do ponto de vista científico, o autor sugere a continuidade das pesquisas com um maior período para a coleta de dados. Propóe que, futuramente, sejam realizados ensaios clínicos buscando a completa validação do RIFLE. Ao mesmo tempo, tentar-se prevenir a LRA, tendo, principalmente, como alvo àqueles que se enquadrarem na classe R. Mesmo que essa não tenha trazido considerável risco de morte quanto às classes I e F, percebeu-se que quase 50\% daqueles pacientes progrediram para as classes mais graves do RIFLE.

\section{Agradecimentos:}

Agradeço a Jorge Dias de Matos pelas orientaçóes, a Thiago Mamoru Sakae pelas revisóes estatísticas, a equipe da UTI do HU-UFSC: Fernando Osni Machado, Rachel Duarte Moritz, Cleiva Mariuza de Oliveira Bueno Aurélio, Francisco José Pinheiro, Luis Antônio Canever, Adelino da Silva, Zurita Estela Martins, Regina Glória Cursio pela colaboração e a dedicaçáo de Acácio Pereira Freitas e de Jorge Augusto Rosso dos Santos.

\section{ABSTRACT}

Objective: To correlate the RIFLE classification with mortality and length of stay both in the intensive care unit and hospital.

Methods: A prospective, observational, longitudinal cohort study, approved by the Institution's Ethics Committee. Data were collected for all patients staying longer than 24 hours in the intensive care unit of Hospital Universitário Polydoro Ernani de São Thiago - Universidade Federal de Santa Catarina from September 2007 to March 2008, followed-up either until discharge or death. Patients were divided in two groups: with or without acute kidney injury. The acute kidney injury group was additionally divided according to the RIFLE and sub-divided according to the maximal score in Risk, Injury of Failure. Loss and End-stage classes were not included in the study. APACHE II and SOFA were also evaluated. The t Student and Chi-Square tests were used. A $P<0.05$ was considered statistically significant.

Results: The sample included 129 patients, 52 (40.3\%) with acute kidney injury according to RIFLE. Patients were more severely ill in this group, with higher APACHE and SOFA scores $(P<0.05)$. Compared to the without kidney injury group, the kidney injury severity caused increased intensive care unity (Risk 25\%; Injury 37.5\%; Failure 62.5\%) and in-hospital (Risk $50 \%$; Injury $37.5 \%$; Failure $62.5 \%)$ mortality, and longer intensive care unit stay $(P<0.05)$.

Conclusion: The RIFLE system, according to the severity class, was a marker for risk of increased intensive care unit and in-hospital mortality, and longer intensive care unit stay. No relationship with in-hospital length of stay was found.

Keywords: Renal insufficiency, acute; Intensive care units; Creatinine; Hospital mortality; Length of stay; APACHE 


\section{REFERÊNCIAS}

1. Uchino S, Bellomo R, Goldsmith D, Bates S, Ronco C. An assessment of the RIFLE criteria for acute renal failure in hospitalized patients. Crit Care Med. 2006;34(7):1913-7.

2. Bellomo R, Ronco C, Kellum JA, Mehta RL, Palevsky P, Acute Dialysis Quality Initiative workgroup. Acute renal failure - definition, outcome measures, animal models, fluid therapy and information technology needs: the Second International Consensus Conference of the Acute Dialysis Quality Iniciative (ADQI) Group. Crit Care. 2004;8(4):R204-12.

3. Metnitz PG, Krenn CG, Steltzer H, Lang T, Ploder J, Lenz $\mathrm{K}$, et al. Effect of acute renal failure requiring renal replacement therapy on outcome in critically ill patients. Crit Care Med. 2002;30(9):2051-8.

4. Chertow GM, Levy EM, Hammermeister KE, Grover F, Daley J. Independent association between acute renal failure and mortality following cardiac surgery. Am J Med. 1998;104(4):343-8.

5. De Mendonça A, Vincent JL, Suter MP, Moreno R, Dearden NM, Antonelli M, et al. Acute renal failure in the ICU: risk factors and outcome evaluated by the SOFA score. Intensive Care Med. 2000;26(7):915-21.

6. Uchino S, Kellum JA, Bellomo R, Doig GS, Morimatsu H, Morgera S, Schetz M, Tan I, Bouman C, Macedo E, Gibney N, Tolwani A, Ronco C; Beginning and Ending Supportive Therapy for the Kidney (BEST Kidney) Investigators. Acute renal failure in critically ill patients: a multinational, multicenter study. JAMA. 2005;294(7):813-8.

7. Bellomo R, Kellum JA, Ronco C. Defining and classifying acute renal failure: from advocacy to consensus and validation of the RIFLE criteria. Intensive Care Med. 2007;33(3):409-13.

8. Bellomo R, Kellum JA, Ronco C. Acute renal failure: time for consensus. Intensive Care Med. 2001;27(11):1685-8.

9. Hoste EA, Clermont G, Kersten A, Venkataraman R, Angus DC, De Bacquer D, Kellum JA. RIFLE criteria for acute kidney injury are associated with hospital mortality in critically ill patients: a cohort analysis. Crit Care. 2006;10(3):R73.

10. Ostermann M, Chang RW. Acute kidney injury in the intensive care unit according to RIFLE. Crit Care Med. 2007;35(8):1837-43; quiz 1852.

11. Kellum JA, Levin N, Bouman C, Lameire N. Developing a consensus classification system for acute renal failure. Curr Opin Crit Care. 2002;8(6):509-14.

12. Vivino G, Antonelli M, Moro ML, Cottini F, Conti G, Bufi M, et al. Risk factors for acute renal failure in trauma patients. Intensive Care Med. 1998;24(8):808-14.

13. Mangano CM, Diamondstone LS, Ramsay JG, Aggarwal A, Herskowitz A, Mangano DT. Renal dysfunction after myocardial revascularization: risk factors, adverse outco- mes, and hospital resourse utilization. The Multicenter Study of Perioperative Ischemia Research Group. Ann Intern Med. 1998;128(3):194-203.

14. Guerin C, Girard R, Selli JM, Perdrix JP, Ayzac L. Initial versus delayed acute renal failure in the intensive care unit. A multicenter prospective epidemiological study. RhôneAlpes Area Study Group on Acute Renal Failure. Am J Respir Crit Care Med. 2000;161(3 Pt 1):872-9. Erratum in: Am J Respir Crit Care Med 2001 Mar;163(3 Pt 1):793-4.

15. Liaño F, Junco E, Pascual J, Madero R, Verde E. The espectrum of acute renal failure in the intensive care unit compared with that seen in other settings. The Madrid Acute Renal Failure Study Group. Kidney Int Suppl. 1998;66:S16-24.

16. Cosentino F, Chaff C, Piedmonte M. Risk factors influencing survival in ICU acute renal failure. Nephrol Dial Transplant. 1994;9 Suppl 4:179-82.

17. Chertow GM, Christiansen CL, Cleary PD, Munro C, Lazarus JM. Prognostic stratification in critically ill patients with acute renal failure requiring dialysis. Arch Intern Med. 1995;155(14):1505-11.

18. Neveu H, Kleinknecht D, Brivet F, Loreit P, Landais P. Prognostic factors in acute renal failure due to sepsis. Results of a prospective multicenter sudy. The French Study Group on Acute Renal Failure. Nephrol Dial Transplant. 1996;11(2):293-9.

19. Mehta RL, Pascual MT, Soroko S, Savage BR, Himmelfarb J, Ikizler TA, Paganini EP, Chertow GM; Program to Improve Care in Acute Renal Disease. Spectrum of acute renal failure in the intensive care unit: the PICARD experience. Kidney Int. 2004;66(4):1613-21.

20. Schaefer JH, Jochimsen F, Keller F, Wegscheider K, Distler A. Outcome prediction of acute renal failure in medical intensive care. Intensive Care Med. 1991;17(1):19-24.

21. Ronco C, Kellum JA, Mehta R. Acute dialysis quality initiative (ADQI). Nephrol Dial Transplant. 2001;16(8):15558.

22. Mehta RL, Kellum JA, Shah SV, Molitoris BA, Ronco C, Warnock DG, Levin A; Acute Kidney Injury Network. Acute Kidney Injury Network: report of an initiative to improve outcomes in acute kidney injury. Crit Care. 2007;11(2):R31.

23. Maccariello E, Soares M, Valente C, Nogueira L, Valença RV, Machado JE, Rocha E. RIFLE classification in patients with acute kidney injury in need of renal replacement therapy. Intensive Care Med. 2007;33(4):597-605.

24. Bell M, Liljestam E, Granath F, Fryckstedt J, Ekbom A, Martling CR. Optimal follow-up time after continuous renal replacement therapy in actual renal failure patients stratified with the RIFLE criteria. Nephrol Dial Transplant. 2005;20(2):354-60.

25. Abosaif NY, Tolba YA, Heap M, Russell J, El Nahas AM. The outcome of acute renal failure in the intensive care 
unit according to RIFLE: model application, sensitivity, and predictability. Am J Kidney Dis. 2005;46(6):1038-48.

26. Kuitunen A, Vento A, Suojaranta-Ylinen R, Pettilä V. Acute renal failure after cardiac surgery: evaluation of the RIFLE classification. Ann Thorac Surg. 2006;81(2):542-6.

27. Lopes JA, Jorge S, Neves FC, Caneira M, da Costa AG, Ferreira AC, Prata MM. An assessment of the RIFLE criteria for acute renal failure in severely burned patients. Nephrol Dial Transplant. 2007;22(1):285.

28. Lopes JA, Fernandes J, Jorge S, Neves J, Antunes F, Prata MM. An assessment of the RIFLE criteria for acute renal failure in critically ill HIV-infected patients. Crit Care. 2007;11(1):401.

29. Lopes JA, Jorge S, Resina C, Santos C, Pereira A, Neves J, et al. Acute renal failure in patients with sepsis. Crit Care. 2007;11(2):411.

30. Barrantes F, Tian J, Vazquez R, Amoateng-Adjepong Y, Manthous CA. Acute kidney injury criteria predict outcomes of critically ill patients. Crit Care Med. 2008;36(5):1397-403.

31. Cruz DN, Bolgan I, Perazella MA, Bonello M, de Cal M, Corradi V, Polanco N, Ocampo C, Nalesso F, Piccinni P, Ronco C; North East Italian Prospective Hospital Renal Outcome Survey on Acute Kidney Injury (NEiPHROSAKI) Investigators. North East Italian Prospective Hospital Renal Outcome Survey on Acute Kidney Injury (NEiPHROS-AKI): targeting the problem with the RIFLE Criteria. Clin J Am Soc Nephrol. 2007;2(3):418-25.
32. Li WX, Chen HD, Wang XW, Zhao S, Chen XK, Zheng Y, Song Y. Predictive value of RIFLE classification on prognosis of critically ill patients with acute kidney injury treated with continuous renal replacement therapy. Chin Med J (Engl). 2009;122(9):1020-5.

33. Bagshaw SM, George C, Bellomo R; ANZICS Database Management Committe. A comparison of the RIFLE and AKIN criteria for acute kidney injury in critically ill patients. Nephrol Dial Transplant. 2008;23(5):1569-74.

34. Joannidis M, Metnitz B, Bauer P, Schusterschitz N, Moreno R, Druml W, Metnitz PG. Acute kidney injury in critically ill patients classified by AKIN versus RIFLE using the SAPS 3 database. Intensive Care Med. 2009;35(10):1692702.

35. Knaus WA, Draper EA, Wagner DP, Zimmerman JE. APACHE II: a severity of disease classification system. Crit Care Med. 1985;13(10):818-29.

36. Vincent JL, Moreno R, Takala J, Willatts S, De Mendonça A, Bruining $\mathrm{H}$, et al. The SOFA (Sepsis-related Organ Failure Assessment) score to describe organ dysfunction/ failure. On behalf of the Working Group on Sepsis-Related Problems of the European Society of Intensive Care Medicine. Intensive Care Med. 1996;22(7):707-10.

37. Levey AS, Bosch JP, Lewis JB, Greene T, Rogers N, Roth D. A more accurate method to estimate glomerular filtration rate from serum creatinine: a new prediction equation. Modification of Diet in Renal Disease Study Group. Ann Intern Med. 1999;130(6):461-70. 